\title{
Permanental ideals of Hankel matrices
}

\author{
Elena Grieco*, Anna Guerrieri ${ }^{\dagger}$ and Irena Swanson ${ }^{\ddagger}$
}

\begin{abstract}
We provide the Gröbner basis and the primary decomposition of the ideals generated by $2 \times 2$ permanents of Hankel matrices.
\end{abstract}

\section{Introduction}

Cauchy [4] and Binet [3] introduced the concept of permanent in 1812 as a special type of alternating symmetric function. The greater part of results on permanents in the nineteenth century consists of identities involving permanents and determinants. Only later the permanents were employed in various fields of applied mathematics, in combinatorics, probability theory, and invariant theory [7. A book that includes the main results about the theory of permanents is 10 .

In this paper we study permanental ideals of Hankel matrices. We focus our attention on the structure of Gröbner basis and primary decomposition of the ideal generated by $2 \times 2$ permanents of Hankel matrices.

The permanent of an $(n \times n)$ matrix $M=\left(a_{i j}\right)$ is defined as

$$
\operatorname{perm}(M)=\sum_{\sigma \in S_{n}} a_{1 \sigma(1)} a_{2 \sigma(2)} \cdots a_{n \sigma(n)} .
$$

Thus, the permanent differs from the determinant only in the lack of minus signs in the expansion. If $M$ is a $u \times v$ matrix with entries in a ring $R$, we denote by $P_{r}(M)$ the ideal of $R$ generated by the $(r \times r)$-subpermanents of $M$. It is particularly interesting when $M$ is a matrix of linear forms in a polynomial ring over a base field $K$. If the field $K$ has characteristic 2 , the permanental ideals equal the determinantal ideals, which are wellunderstood [5], 11]. Thus, from now on, we assume that the characteristic is different from 2 .

\footnotetext{
*Dipartimento di Matematica Pura e Applicata - Università degli Studi dell'Aquila Via Vetoio snc, 67010 COPPITO (AQ) Italy - grieco@univaq.it.

${ }^{\dagger}$ Dipartimento di Matematica Pura e Applicata - Università degli Studi dell'Aquila Via Vetoio snc, 67010 COPPITO (AQ) Italy - guerrran@univaq.it.

${ }^{\ddagger}$ Department of Mathematics - Reed College - 3203 SE Woodstock Blvd Portland, OR 97202 - iswanson@reed.edu.
} 
Laubenbacher and Swanson [9] studied the case of generic matrices finding the primary decomposition of $P_{2}(M)$. Recently Kirkup [8] gives some indication on associated primes of the ideal generated by $3 \times 3$ permanents of a generic matrix (not complete list).

We analyze the case of Hankel matrices, finding profoundly different results from 9 . Let $K$ be a field, $m, n, r$ positive integers, and $x_{i}$ variables over $K$ with $1 \leq i \leq m+n-1$. Let $R=K\left[x_{i} \mid 1 \leq i \leq m+n-1\right]$ be the polynomial ring over the previous variables, and let $M$ be an $m \times n$ Hankel matrix in $R$, with $m \leq n$

$$
M=\left[\begin{array}{cccc}
x_{1} & x_{2} & \cdots & x_{n} \\
x_{2} & x_{3} & \cdots & x_{n+1} \\
\vdots & \vdots & & \vdots \\
x_{m} & x_{m+1} & \cdots & x_{n+m-1}
\end{array}\right]
$$

As $P_{2}(M)$ for a $2 \times 2$ Hankel matrix is a prime ideal, in the rest of paper we assume that $m+n>4$.

In Section 2 we analyze the structure of a Gröbner basis, in some cases reduced, of the ideal $P_{2}(M)$. To simplify the work we start with some preliminary Lemmas about the existence of particular monomials in the ideal $P_{2}(M)$, (Lemmas 2.1 and 2.2), about the reduction of monomials of degree 2 and 3, (Lemma 2.4), and about the $S$-polynomial of two permanents, (Lemma 2.5) with respect to the set $H$ of all permanents. In the Theorem 2.7 we find the reduced Gröbner basis for $P_{2}(M)$ of a $2 \times n$ Hankel matrix, and in the Theorem 2.10 we provide a Gröbner basis for $P_{2}(M)$ of a $m \times n$ Hankel matrix with $m \geq 3$ and $n \geq 5$. We are left with the cases $3 \times 3$, $3 \times 4$ and $4 \times 4$. Their Gröbner bases are different from the general case, we deal them respectively in the Proposition 2.8, (3×3 matrix), and in the Proposition 2.9, $(3 \times 4$ and $4 \times 4$ matrices $)$. We note that for a Hankel matrix, the Gröbner basis of $P_{2}(M)$ depends strongly on the shape of matrix, whereas if $M$ is a $m \times n$ generic matrix there exists a unique pattern for any $m, n$, see [9].

In Section 3 we show that the permanental ideal $P_{2}(M)$ has exactly two minimal primes, see Proposition [3.1, and that the structure of these primes does not depend on the shape of matrix. This is exactly the opposite of the situation described in 9]. In Proposition 3.2 we provide also the minimal components of $P_{2}(M)$, which are different from the minimal primes. This is in contrast with the results for generic matrices in [9. In Section 4, 5] and 6] we provide the full primary decomposition of $P_{2}(M)$. Again we need to separate cases. In Section 4 we give a primary decomposition, probably redundant, of the ideal $P_{2}(M)$. With respect to [9] the first difference is about the number and the structure of minimal primes, the second difference is about the embedded component. We show that, except for few cases, there exists exactly one embedded component and the associated prime is the maximal ideal. It is important to underline that, if $M$ is a generic matrix 
then the embedded component exists if and only if $m, n \geq 3$, whereas if $M$ is a Hankel matrix the existence of the embedded component depends on the shape of matrix, as we show in the Sections 5 and 6 .

\section{Gröbner Bases}

We first need to recall some results of Laubenbacher and Swanson 9] that are true also in the case of Hankel matrices. We omit the proofs that are identical to those of [9].

Lemma 2.1. The ideal $P_{2}(M)$ contains all the products of three entries of $M$, taken from three distinct columns and two distinct rows, or from two distinct columns and three distinct rows.

Lemma 2.2. If $m, n \geq 3, P_{2}(M)$ contains all monomials of the form $x_{i_{1} j_{1}}^{e_{1}} x_{i_{2} j_{2}}^{e_{2}} x_{i_{3} j_{3}}^{e_{3}}$ with distinct $i_{1}, i_{2}, i_{3}$, distinct $j_{1}, j_{2}, j_{3}$, and where $e_{1}, e_{2}, e_{3}$ are positive integers which sum to 4 .

Observation 2.3. In our case $x_{i j}$ means $x_{i+j-1}$.

We start now the work to find a Gröbner basis for $P_{2}(M)$ with respect to a lexicographic monomial order, with $x_{1}>x_{2}>\cdots>x_{m+n-1}$.

Lemma 2.4. Let $M$ be $a m \times n$ Hankel matrix as in (11) and let $H$ be the set of all permanents of $M$. Define the lexicographic ordering of monomials, with $x_{1}>x_{2}>\cdots>x_{m+n-1}$. Then for $i, j, k=1, \ldots, m+n-1$

1. the reduction of $x_{i} x_{j}$ with respect to $H$ is

(a) $(-1)^{j-1-\frac{i+j}{2}} x_{\frac{i+j}{2}}^{2}$, if $i+j \equiv 0 \bmod 2$,

(b) $(-1)^{j-1-\frac{i+j+1}{2}} x_{\frac{i+j-1}{2}} x_{\frac{i+j+1}{2}}$, if $i+j \equiv 1 \bmod 2$;

2. the reduction of $x_{i} x_{j} x_{k}$ with respect to $H$ is

(a) $\pm x_{\frac{i+j+k}{3}}^{3}$, if $i+j+k \equiv 0 \bmod 3$,

(b) $\pm x_{\frac{i+j+k-1}{3}}^{2} x_{\frac{i+j+k+2}{3}}$, if $i+j+k \equiv 1 \bmod 3$,

(c) $\pm x_{\frac{i+j+k-2}{3}} x_{\frac{i+j+k+1}{3}}^{2}$ if $i+j+k \equiv 2 \bmod 3$.

Proof. In general case we can assume that $i \leq j \leq k$.

1. It is clear that if $i=j$ then $i+j \equiv 0 \bmod 2$ and $x_{i} x_{j} \equiv x_{i}^{2}$, and if $j=i+1$ then $i+j \equiv 1 \bmod 2$ and $x_{i} x_{j} \equiv x_{i} x_{i+1}$. Suppose now that $j>i+1$. By induction assumption we have

$x_{i} x_{j} \equiv-x_{i+1} x_{j-1} \equiv- \begin{cases}(-1)^{j-2-\frac{i+j}{2}} x_{\frac{i+j}{2}}^{2} & \text { if } i+j \equiv 0 \bmod 2 \\ (-1)^{j-2-\frac{i+j+1}{2}} x_{\frac{i+j-1}{2}} x_{\frac{i+j+1}{2}} & \text { if } i+j \equiv 1 \bmod 2\end{cases}$

and so, we are done. 
2. Clearly if $k=i$ then $i+j+k \equiv 0 \bmod 3$ and $x_{i} x_{j} x_{k}=x_{i}^{3}$. Suppose $k=i+1$. Then we have two cases: if $j=i$ then $i+j+k \equiv 1 \bmod 3$ and $x_{i}^{2} x_{i+1}$, and if $j=k$ then $i+j+k \equiv 2 \bmod 3$ and $x_{i} x_{i+1}^{2}$. Suppose now that $k>i+1$. Then

$$
x_{i} x_{j} x_{k} \equiv-x_{i+1} x_{j} x_{k-1},
$$

and for all $j=i, \ldots, k$ the difference between $\max \{i+1, j, k-1\}$ and $\min \{i+1, j, k-1\}$ is strictly smaller than $k-i$, so by induction assumption on $k-i$ we have

$$
x_{i} x_{j} x_{k} \equiv-x_{i+1} x_{j} x_{k-1} \equiv \begin{cases} \pm x_{\frac{i+j+k}{3}}^{3} & \text { if } i+j+k \equiv 0 \bmod 3 \\ \pm x_{\frac{i+j+k-1}{3}}^{2} x_{\frac{i+j+k+2}{3}} & \text { if } i+j+k \equiv 1 \bmod 3 \\ \pm x_{\frac{i+j+k-2}{3}}^{2} x_{\frac{i+j+k+1}{3}}^{2} & \text { if } i+j+k \equiv 2 \bmod 3\end{cases}
$$

and so, we are done.

Lemma 2.5. Let $M$ be an $m \times n$ Hankel matrix as in (1). Define the lexicographic ordering of monomials, with $x_{1}>x_{2}>\cdots>x_{m+n-1}$. Let $f$, $g$ be two permanents of $M$ with distinct leading terms. Then $S(f, g)$ either trivially reduces to zero or it is a binomial whose terms $x_{a} x_{b} x_{c}$ satisfy

$$
7 \leq a+b+c \leq 3 m+3 n-7 .
$$

Proof. By hypothesis on $f$ and $g$, their leading term can have only one factor in common. Let

$$
\begin{gathered}
f=x_{i} x_{i+s+t}+x_{i+s} x_{i+t}, \\
g=x_{j} x_{j+s^{\prime}+t^{\prime}}+x_{j+s^{\prime}} x_{j+t^{\prime}} .
\end{gathered}
$$

First suppose that $i=j$. Then

$$
S(f, g)=x_{i+s} x_{i+t} x_{j+s^{\prime}+t^{\prime}}-x_{j+s^{\prime}} x_{j+t^{\prime}} x_{i+s+t} .
$$

We can suppose also that $i+s+t<j+s^{\prime}+t^{\prime}$, so we have

$$
\begin{gathered}
3 \leq i+s+t \leq m+n-2, \\
4 \leq j+s^{\prime}+t^{\prime} \leq m+n-1, \\
1 \leq i, j \leq m+n-4 .
\end{gathered}
$$

In this case the sum of indices $a+b+c$ is the same for both terms. It is simple to see that

$1+3+4 \leq i+(s+i+t)+\left(j+s^{\prime}+t^{\prime}\right) \leq m+n-4+m+n-2+m+n-1$.

So we have

$$
8 \leq a+b+c \leq 3 m+3 n-7 .
$$


Now we suppose that $i=j+s^{\prime}+t^{\prime}$. Then

$$
S(f, g)=x_{j} x_{i+s} x_{i+t}-x_{j+s^{\prime}} x_{j+t^{\prime}} x_{i+s+t} .
$$

Clearly we have

$$
\begin{gathered}
1 \leq j \leq i-s^{\prime}-t^{\prime} \leq(m+n-3)-1-1=m+n-5, \\
3 \leq i=j+s^{\prime}+t^{\prime} \leq m+n-3, \\
5=3+1+1 \leq\left(j+s^{\prime}+t^{\prime}\right)+s+t=i+s+t \leq m+n-1 .
\end{gathered}
$$

Also in this case the sum of indices $a+b+c$ is the same for both terms of $S(f, g)$ and it satisfies the following relation:

$$
1+3+5 \leq(j)+(i)+(s+i+t) \leq m+n-5+m+n-3+m+n-1 .
$$

So we have

$$
9 \leq a+b+c \leq 3 m+3 n-9 .
$$

Finally, we suppose that $i+s+t=j+s^{\prime}+t^{\prime}$. Then

$$
S(f, g)=x_{i} x_{j+s^{\prime}} x_{j+t^{\prime}}+x_{j} x_{i+s} x_{i+t} .
$$

We can suppose that $i<j$. So we have

$$
\begin{gathered}
1 \leq i \leq m+n-4, \\
2 \leq j \leq m+n-3 \\
4=j+s^{\prime}+t^{\prime} \leq i+s+t \leq m+n-1 .
\end{gathered}
$$

Clearly, also in this case the sum of indices $a+b+c$ is equal for both of terms of $S(f, g)$ and it satisfies

$$
1+2+4 \leq(i)+(j)+\left(s^{\prime}+j+t^{\prime}\right) \leq m+n-4+m+n-3-m+n-1 .
$$

So we have

$$
7 \leq a+b+c \leq 3 m+3 n-8
$$

Corollary 2.6. Under assumptions of Lemma 2.5, if a set $H$ contains all the permanents of $M, x_{3}^{3}, x_{4}^{3}, \ldots, x_{m+n-3}^{3}, x_{2}^{2} x_{3}, x_{3}^{2} x_{4}, \ldots, x_{m+n-3}^{2} x_{m+n-2}$, $x_{2} x_{3}^{2}, x_{3} x_{4}^{2}, \ldots, x_{m+n-3} x_{m+n-2}^{2}$, then $S(f, g)$ reduces to zero with respect to $H$. 
Proof. It is sufficient to prove that each of the terms of $S(f, g)$ reduces to a multiple of some monomial of $H$. By Lemma 2.5 it is sufficient to prove that whenever $i, j, k$ are positive integers with $7 \leq i+j+k \leq 3 m+3 n-7$, then $x_{i} x_{j} x_{k}$ reduces to zero with respect to $H$. By Lemma $2.4 x_{i} x_{j} x_{k}$ reduces, with respect to permanents, to

$$
\begin{array}{ll} 
\pm x_{\frac{i+j+k}{3}}^{3}, & \text { if } i+j+k \equiv 0 \bmod 3, \\
\pm x_{\frac{i+j+k-1}{3}}^{2} x_{\frac{i+j+k+2}{3}}, & \text { if } i+j+k \equiv 1 \bmod 3, \\
\pm x_{\frac{i+j+k-2}{3}}^{2} x_{\frac{i+j+k+1}{3}}^{2} & \text { if } i+j+k \equiv 2 \bmod 3 .
\end{array}
$$

But the relation $7 \leq i+j+k \leq 3 m+3 n-7$ implies that, up to sign, these reduced monomials are in the set $x_{3}^{3}, x_{4}^{3}, \ldots, x_{m+n-3}^{3}, x_{2}^{2} x_{3}, x_{3}^{2} x_{4}, \ldots$, $x_{m+n-3}^{2} x_{m+n-2}, x_{2} x_{3}^{2}, x_{3} x_{4}^{2} \ldots, x_{m+n-3} x_{m+n-2}^{2}$, which are all elements of $H$.

Theorem 2.7. Let $M$ be a $2 \times n$ Hankel matrix, with $n \geq 3$, and let $G$ be the following set of polynomials:

1. the permanents $x_{i} x_{i+t+1}+x_{i+1} x_{i+t}, i=1, \ldots, n-1, t=1, \ldots, n-i$;

2. $x_{i}^{2} x_{i+1}, i=2, \ldots, n-1$;

3. $x_{i} x_{i+1}^{2}, i=2, \ldots, n-1$;

4. $x_{i}^{3}, i=3, \ldots, n-1$;

5. $x_{2}^{4}, x_{n}^{4}$.

Then $G$ is a minimal reduced Gröbner basis for $P_{2}(M)$ with respect to the lexicographic ordering of monomials, with $x_{1}>x_{2}>\cdots>x_{n+1}$.

Proof. First of all we observe that in case $n=3$ the set 4 is empty. Clearly $P_{2}(M)$ contains all elements of type 1 . By Lemma 2.1 it is clear that $P_{2}(M)$ contains all elements of type 2 and 3 of $G$. It is easy to prove that also the monomials of type 4 and 5 are in $P_{2}(M)$. In fact, as the Lemma 2.1 assures that for all $i=3, \ldots, n-1, x_{i-1} x_{i} x_{i+1}, x_{2}^{2} x_{3}$ and $x_{n-1} x_{n}^{2}$ are in $P_{2}(M)$, we have

$$
\begin{gathered}
x_{i}^{3}=x_{i}\left(x_{i-1} x_{i+1}+x_{i}^{2}\right)-x_{i-1} x_{i} x_{i+1} \in P_{2}(M), \\
x_{2}^{4}=x_{2}^{2}\left(x_{1} x_{3}+x_{2}^{2}\right)-x_{1} x_{2}^{2} x_{3} \in P_{2}(M), \\
x_{n}^{4}=x_{n}^{2}\left(x_{n-1} x_{n+1}+x_{n}^{2}\right)-x_{n-1} x_{n}^{2} x_{n+1} \in P_{2}(M) .
\end{gathered}
$$

It is easy to see also that $G$ is a reduced and minimal generating set for $P_{2}(M)$. Thus it is sufficient to prove that the $S$-polynomials of elements of $G$ reduce to zero with respect to $G$. As the $S$-polynomial of two monomials reduces to zero, it remains to show that $S(f, g)$ reduces to zero when $f$ is a 
permanent.

First consider $f, g$ two permanents

$$
\begin{aligned}
& f=x_{i} x_{i+s+1}+x_{i+1} x_{i+s}, \\
& g=x_{j} x_{j+t+1}+x_{j+1} x_{j+t},
\end{aligned}
$$

with $s \leq t$. Clearly $x_{i} x_{i+s+1}$ and $x_{j} x_{j+t+1}$ are respectively the leading terms of $f$ and $g$. If $i n(f)$ and $i n(g)$ have no factor in common then $S(f, g)$ reduces to zero. If instead $i n(f)$ and $i n(g)$ have exactly one variable in common, then $S(f, g)$ reduces to zero with respect to $G$, by Corollary 2.6.

Now, we see what happens when $f$ is a permanent and $g$ is a monomial. We first consider $g$ a monomial of type 2

$$
\begin{gathered}
f=x_{i} x_{i+s+1}+x_{i+1} x_{i+s}, \\
g=x_{j}^{2} x_{j+1} .
\end{gathered}
$$

If $i=j$, then

$$
S(f, g)=-x_{i} x_{i+1}^{2} x_{i+s} .
$$

As $i=j=2, \ldots, n-1$, it reduces to zero with respect to a monomial of type 3 .

If $i=j+1$, then

$$
S(f, g)=-x_{i-1}^{2} x_{i+1} x_{i+s}
$$

reduces to $x_{i-1} x_{i}^{2} x_{i+t}$ with respect to $x_{i-1} x_{i+1}+x_{i}^{2}$. As $i=j+1=3, \ldots, n$, then it reduces to zero with respect to a monomial of type 3 .

Suppose $i+s+1=j$. Clearly if $j=2$ then $i+s+t \neq j$. So $i+s+t=j=$ $3, \ldots, n-1$, and

$$
S(f, g)=-x_{i+1} x_{i+s} x_{i+s+1} x_{i+s+2} .
$$

But $\alpha=x_{i+s} x_{i+s+2}+x_{i+s+1}^{2}$ is a permanent, so $S(f, g)$ reduces to $x_{i+1} x_{i+s+1}^{3}$ with respect to $\alpha$, which reduces to zero with respect to a monomial of type 4 .

At the end we suppose that $i+s=j=2, \ldots, n-1$ and

$$
S(f, g)=x_{i+1} x_{i+s}^{3} .
$$

If $i+s=2$ then $S(f, g)=x_{2}^{4}$ which reduces to zero. If $i+s=3, \ldots n-1$, $S(f, g)$ reduces to zero with respect to a monomial of type 4 .

In the same way we can show that $S(f, g)$ reduces to zero when $g$ is a monomial of type 3 .

Now we suppose that $g$ is a monomial of type $4, g=x_{j}^{3}$.

If $i=j=2, \ldots, n-1$, then $S(f, g)=x_{i}^{2} x_{i+1}^{2} x_{i+s}$ reduces to zero with respect to a monomial of type 2 or 3 .

If $i+s+1=j$, then $S(f, g)=x_{i+1} x_{i+s} x_{i+s+1}^{2}$. It is clear that if $j=2$ then 
$i+s+1 \neq j$. So $i+s+1=j=3, \ldots, n-1$ and $S(f, g)$ reduces to zero with respect to a monomial of type 3 .

At the end, we see what about $f$ permanent and $g=x_{2}^{4}$ or $g=x_{n}^{4}$.

If $i \neq 2, S\left(f, x_{2}^{4}\right)=0$ and if $i=2, S\left(f, x_{2}^{4}\right)=x_{2}^{3} x_{3} x_{2+t}$ which reduces to zero.

If $i+t+1 \neq n, S\left(f, x_{n}^{4}\right)=0$ and if $i+t+1=n, S\left(f, x_{n}^{4}\right)=x_{i+1} x_{n-1} x_{n}^{3}$ which reduces to zero.

Proposition 2.8. Let $M$ be a $3 \times 3$ Hankel matrix and let $G$ be the following set of polynomials:

1. the permanents $x_{i} x_{i+s+t}+x_{i+s} x_{i+t}, i=1,2,3, s, t=1,2$ with $i+s+t=$ $3,4,5$;

2. $x_{2}^{2} x_{3}, x_{2} x_{3}^{2}, x_{3}^{2} x_{4}, x_{3} x_{4}^{2}$;

3. $x_{2}^{4}, x_{3}^{4}, x_{4}^{4}$.

Then $G$ is a minimal reduced Gröbner basis for $P_{2}(M)$ with respect to the lexicographic ordering of monomials with $x_{1}>x_{2}>\cdots>x_{5}$.

Proof. Clearly $P_{2}(M)$ contains all elements of type 1. By Lemma 2.1. it is clear that $P_{2}(M)$ contains all elements of type 2 of $G$. Furthermore, by Lemma 2.1 $x_{2}^{2} x_{3}, x_{3}^{2} x_{5}, x_{3} x_{4}^{2}$ are in $P_{2}(M)$, then

$$
\begin{aligned}
& x_{2}^{4}=x_{2}^{2}\left(x_{1} x_{3}+x_{2}^{2}\right)-x_{1} x_{2}^{2} x_{3}, \\
& x_{3}^{4}=x_{3}^{2}\left(x_{1} x_{5}+x_{3}^{2}\right)-x_{1} x_{3}^{2} x_{5}, \\
& x_{4}^{4}=x_{4}^{2}\left(x_{3} x_{5}+x_{4}^{2}\right)-x_{3} x_{4}^{2} x_{5},
\end{aligned}
$$

are in $P_{2}(M)$, so that also elements of type 3 of $G$ are in $P_{2}(M)$. It is easy to see also that $G$ is a reduced and a minimal generating set for $P_{2}(M)$. Therefore it is sufficient to prove that the $S$-polynomials of elements of $G$ reduce to zero with respect to $G$. As the $S$-polynomial of two monomials reduces to zero, it remains to show that $S(f, g)$ reduces to zero when at least one of $f$ and $g$ is a permanent. If $i n(f)$ and $i n(g)$ have no variables in common, then $S(f, g)$ always reduces to zero. In particular,

$$
\begin{gathered}
S\left(x_{1} x_{3}+x_{2}^{2}, x_{2} x_{4}+x_{3}^{2}\right), S\left(x_{1} x_{3}+x_{2}^{2}, x_{2} x_{5}+x_{3} x_{4}\right), S\left(x_{1} x_{3}+x_{2}^{2}, x_{2}^{4}\right), \\
S\left(x_{1} x_{3}+x_{2}^{2}, x_{4}^{4}\right), S\left(x_{1} x_{4}+x_{2} x_{3}, x_{2} x_{5}+x_{3}^{2}\right), S\left(x_{1} x_{4}+x_{2} x_{3}, x_{3} x_{5}+x_{4}^{2}\right), \\
S\left(x_{1} x_{4}+x_{2} x_{3}, x_{2}^{2} x_{3}\right), S\left(x_{1} x_{4}+x_{2} x_{3}, x_{2} x_{3}^{2}\right), S\left(x_{1} x_{4}+x_{2} x_{3}, x_{2}^{4}\right), \\
S\left(x_{1} x_{4}+x_{2} x_{3}, x_{3}^{4}\right), S\left(x_{1} x_{5}+x_{3}^{2}, x_{2} x_{4}+x_{3}^{2}\right), \\
S\left(x_{1} x_{5}+x_{3}^{2}, g\right) \text { for all monomials } g \in G \\
S\left(x_{2} x_{4}+x_{3}^{2}, x_{3} x_{5}+x_{4}^{2}\right), S\left(x_{2} x_{4}+x_{3}^{2}, x_{3}^{4}\right), S\left(x_{2} x_{5}+x_{3} x_{4}, x_{3}^{2} x_{4}\right),
\end{gathered}
$$




$$
\begin{gathered}
S\left(x_{2} x_{5}+x_{3} x_{4}, x_{3} x_{4}^{2}\right), S\left(x_{2} x_{5}+x_{3} x_{4}, x_{3}^{4}\right), S\left(x_{2} x_{5}+x_{3} x_{4}, x_{4}^{4}\right), \\
S\left(x_{3} x_{5}+x_{4}^{2}, x_{2}^{4}\right), S\left(x_{3} x_{5}+x_{4}^{2}, x_{4}^{4}\right) \longrightarrow 0 .
\end{gathered}
$$

If $f$ and $g$ are permanents and $\operatorname{lcm}(i n(f), i n(g))=x_{a} x_{b} x_{c}$, with $a+b+c$ not a multiple of 3 , then as in the proof of Corollary [2.6, $S(f, g)$ reduces to zero. In particular,

$$
\begin{gathered}
S\left(x_{1} x_{3}+x_{2}^{2}, x_{1} x_{4}+x_{2} x_{3}\right), S\left(x_{1} x_{4}+x_{2} x_{3}, x_{1} x_{5}+x_{3}^{2}\right), \\
S\left(x_{1} x_{4}+x_{2} x_{3}, x_{2} x_{4}+x_{3}^{2}\right), S\left(x_{1} x_{5}+x_{3}^{2}, x_{2} x_{5}+x_{3} x_{4}\right), \\
S\left(x_{2} x_{4}+x_{3}^{2}, x_{2} x_{5}+x_{3} x_{4}\right), S\left(x_{2} x_{5}+x_{3} x_{4}, x_{3} x_{5}+x_{4}^{2}\right) \longrightarrow 0 .
\end{gathered}
$$

And here is the remainder of the reductions of $S$-polynomials:

$$
\begin{aligned}
& S\left(x_{1} x_{3}+x_{2}^{2}, x_{1} x_{5}+x_{3}^{2}\right)=x_{2}^{2} x_{5}-x_{3}^{3} \stackrel{x_{2} x_{5}+x_{3} x_{4}}{\longrightarrow}-x_{3}\left(x_{2} x_{4}+x_{3}^{2}\right) \longrightarrow 0, \\
& S\left(x_{1} x_{3}+x_{2}^{2}, x_{3} x_{5}+x_{4}^{2}\right)=x_{2}^{2} x_{5}-x_{1} x_{4}^{2} \stackrel{x_{2} x_{5}+x_{3} x_{4}}{\longrightarrow}-x_{4}\left(x_{1} x_{4}+x_{2} x_{3}\right) \longrightarrow 0, \\
& S\left(x_{1} x_{3}+x_{2}^{2}, x_{2}^{2} x_{3}\right)=x_{2}^{4} \longrightarrow 0, \\
& S\left(x_{1} x_{3}+x_{2}^{2}, x_{2} x_{3}^{3}\right)=x_{2}^{3} x_{3} \longrightarrow 0, \\
& S\left(x_{1} x_{3}+x_{2}^{2}, x_{3}^{2} x_{4}\right)=x_{2}^{2} x_{3} x_{4} \longrightarrow 0, \\
& S\left(x_{1} x_{3}+x_{2}^{2}, x_{3} x_{4}^{2}\right)=x_{2}^{2} x_{4}^{4} \stackrel{x_{2} x_{4}+x_{3}^{2}}{\longrightarrow} x_{3}^{4} \longrightarrow 0, \\
& S\left(x_{1} x_{3}+x_{2}^{2}, x_{3}^{4}\right)=x_{2}^{2} x_{3}^{3} \longrightarrow 0, \\
& S\left(x_{1} x_{4}+x_{2} x_{3}, x_{3}^{2} x_{4}\right)=x_{2} x_{3}^{3} \longrightarrow 0, \\
& S\left(x_{1} x_{4}+x_{2} x_{3}, x_{3} x_{4}^{2}\right)=x_{2} x_{3}^{2} x_{4} \longrightarrow 0, \\
& S\left(x_{1} x_{4}+x_{2} x_{3}, x_{4}^{4}\right)=x_{2} x_{3} x_{4}^{3} \longrightarrow 0, \\
& S\left(x_{1} x_{5}+x_{3}^{2}, x_{3} x_{5}+x_{4}^{2}\right)=-x_{1} x_{4}^{2}+x_{3}^{3} \stackrel{x_{1} x_{4}+x_{2} x_{3}}{\longrightarrow} x_{3}\left(x_{2} x_{4}+x_{3}^{2}\right) \longrightarrow 0, \\
& S\left(x_{2} x_{4}+x_{3}^{2}, x_{2}^{2} x_{3}\right)=x_{2} x_{3}^{3} \longrightarrow 0, \\
& S\left(x_{2} x_{4}+x_{3}^{2}, x_{2} x_{3}^{2}\right)=x_{3}^{4} \longrightarrow 0, \\
& S\left(x_{2} x_{4}+x_{3}^{2}, x_{3}^{2} x_{4}\right)=x_{3}^{4} \longrightarrow 0, \\
& S\left(x_{2} x_{4}+x_{3}^{2}, x_{3} x_{4}^{2}\right)=x_{3}^{3} x_{4} \longrightarrow 0, \\
& S\left(x_{2} x_{4}+x_{3}^{2}, x_{2}^{4}\right)=x_{2}^{3} x_{3}^{2} \longrightarrow 0, \\
& S\left(x_{2} x_{4}+x_{3}^{2}, x_{4}^{4}\right)=x_{3}^{2} x_{4}^{3} \longrightarrow 0, \\
& S\left(x_{2} x_{5}+x_{3} x_{4}, x_{2}^{2} x_{3}\right)=x_{2} x_{3}^{2} x_{4} \longrightarrow 0, \\
& S\left(x_{2} x_{5}+x_{3} x_{4}, x_{2} x_{3}^{2}\right)=x_{3}^{3} x_{4} \longrightarrow 0, \\
& S\left(x_{2} x_{5}+x_{3} x_{4}, x_{2}^{4}\right)=x_{2}^{3} x_{3} x_{4} \longrightarrow 0,
\end{aligned}
$$




$$
\begin{gathered}
S\left(x_{3} x_{5}+x_{4}^{2}, x_{2}^{2} x_{3}\right)=x_{2}^{2} x_{4}^{4} \stackrel{x_{2} x_{4}+x_{3}^{2}}{\longrightarrow}-x_{2} x_{3}^{2} x_{4} \longrightarrow 0, \\
S\left(x_{3} x_{5}+x_{4}^{2}, x_{2} x_{3}^{2}\right)=x_{2} x_{3} x_{4}^{2} \longrightarrow 0 \\
S\left(x_{3} x_{5}+x_{4}^{2}, x_{3}^{3} x_{4}\right)=x_{3} x_{4}^{3} \longrightarrow 0 \\
S\left(x_{3} x_{5}+x_{4}^{2}, x_{3} x_{4}^{2}\right)=x_{4}^{4} \longrightarrow 0 \\
S\left(x_{3} x_{5}+x_{4}^{2}, x_{3}^{4}\right)=x_{3}^{3} x_{4}^{2} \longrightarrow 0
\end{gathered}
$$

Proposition 2.9. Let $M$ be a $m \times n$ Hankel matrix, where $(m, n)=(3,4)$ or $(m, n)=(4,4)$ and let $G$ be the following set of polynomials:

1. the permanents $x_{i} x_{i+s+t}+x_{i+s} x_{i+t}, i=1, \ldots, m+n-3, s=1, \ldots, m-$ $1, t=1, \ldots n-1$ with $i+s+t=3, \ldots, m+n-1$;

2. $x_{2}^{2} x_{3}, x_{m+n-3} x_{m+n-2}^{2}$;

3. $x_{3}^{2}, \ldots, x_{m+n-3}^{2}$;

4. $x_{2}^{4}, x_{m+n-2}^{4}$.

Then $G$ is a minimal reduced Gröbner basis for $P_{2}(M)$ with respect to the lexicographic ordering of monomials, with $x_{1}>x_{2}>\cdots>x_{m+n-1}$.

Proof. As the proof of Proposition 2.9 is similar to that of Proposition 2.8 we omit it.

Theorem 2.10. Let $M$ be an $m \times n$ Hankel matrix with $m \geq 3$ and $n \geq 5$, and let $G$ be the following set of polynomials:

1. the permanents $x_{i} x_{i+s+t}+x_{i+s} x_{i+t}, i=1, \ldots, m+n-3, s=1, \ldots, m-$ $1, t=1, \ldots, n-1$ with $i+s+t=3, \ldots, m+n-1$;

2. $x_{i} x_{i+1}, i=3, \ldots, m+n-4$;

3. $x_{2}^{2} x_{3}, x_{m+n-3} x_{m+n-2}^{2}$;

4. $x_{i}^{2}, i=3, \ldots, m+n-3$;

5. $x_{2}^{4}, x_{m+n-2}^{4}$.

Then $G$ is a Gröbner basis for $P_{2}(M)$ with respect to the lexicographic ordering of monomials with $x_{1}>x_{2}>\cdots>x_{m+n-1}$. 
Proof. First of all we show that $P_{2}(M)$ contains $G$. Clearly $P_{2}(M)$ contains all elements of type 1 , and by Lemma 2.1 it is clear that $P_{2}(M)$ contains both elements of type 3 of $G$. It remains to prove that also the monomials of type 2,4 and 5 are in $P_{2}(M)$. We can consider the following submatrix of $M$

$$
\left[\begin{array}{ccccc}
x_{i-2} & x_{i-1} & x_{i} & x_{i+1} & x_{i+2} \\
x_{i-1} & x_{i} & x_{i+1} & x_{i+2} & x_{i+3} \\
x_{i} & x_{i+1} & x_{i+2} & x_{i+3} & x_{i+4}
\end{array}\right] .
$$

Then, for all $i=3, \ldots, m+n-4$, we have

$2 x_{i} x_{i+1}=\left(x_{i-1} x_{i+2}+x_{i} x_{i+1}\right)+\left(x_{i-2} x_{i+3}+x_{i} x_{i+1}\right)-\left(x_{i-2} x_{i+3}+x_{i-1} x_{i+2}\right)$.

Moreover, for all $i=3, \ldots, m+n-3$ we have

$$
2 x_{i}^{2}=\left(x_{i-1} x_{i+1}+x_{i}^{2}\right)+\left(x_{i-2} x_{i+2}+x_{i}^{2}\right)-\left(x_{i-2} x_{i+2}+x_{i-1} x_{i+1}\right) .
$$

This proves that the monomials of type 2 and 4 are in $P_{2}(M)$. Finally

$$
\begin{gathered}
x_{2}^{4}=x_{2}^{2}\left(x_{1} x_{3}+x_{2}^{2}\right)-x_{1} x_{2}^{2} x_{3} \\
x_{m+n-2}^{4}=x_{m+n-2}^{2}\left(x_{m+n-3} x_{m+n-1}+x_{m+n-2}^{2}\right)-x_{m+n-3} x_{m+n-2}^{2} x_{m+n-1} .
\end{gathered}
$$

Because $x_{2}^{2} x_{3}$ and $x_{m+n-2}^{2} x_{m+n-1}$ lie in $P_{2}(M)$ by Lemma 2.1 we conclude that $G$ is in $P_{2}(M)$. Now we show that the $S$-polynomials of elements of $G$ reduce to zero with respect to $G$. As the $S$-polynomial of two monomials reduces to zero, it remains to show that $S(f, g)$ reduces to zero when at least one of $f$ and $g$ is a permanent. First of all we consider the case in which both of them are permanents. Let

$$
\begin{gathered}
f=x_{i} x_{i+s+t}+x_{i+s} x_{i+t}, \\
g=x_{j} x_{j+s^{\prime}+t^{\prime}}+x_{j+s^{\prime}} x_{j+t^{\prime}},
\end{gathered}
$$

with $i, j=1, \ldots, m+n-3, s=1, \ldots, m-1, s^{\prime}=1, \ldots, m-1, t=1, \ldots, n-1$, $t^{\prime}=1, \ldots, n-1$, with $i+s+t, j+s^{\prime}+t^{\prime} \leq m+n-1$. We can suppose also that $s \leq t$ and $s^{\prime} \leq t^{\prime}$. Clearly inf $=x_{i} x_{i+s+t}$ and $i n(g)=x_{j} x_{j+s^{\prime}+t^{\prime}}$. If in $(f)$ and in $(g)$ have no factor in common then $S(f, g)$ reduces to zero. So we suppose that $i n(f)$ and $i n(g)$ have at least one variable in common. First of all we suppose that $i n(f)=i n(g)$ and $f \neq g$, so $i=j$ and $s+t=s^{\prime}+t^{\prime}$ but $s \neq s^{\prime}$ and $t \neq t^{\prime}$. Suppose $s^{\prime}<s$, then there exists $r=1, \ldots, s-1$ such that $s^{\prime}=s-r$ and $t^{\prime}=t+r$, and so we have

$$
S(f, g)=x_{i+s-r} x_{i+t+r}-x_{i+s} x_{i+t} .
$$

But we can consider the permanent $\alpha=x_{i+s-r} x_{i+t+r}+x_{i+s} x_{i+t}$ and so $S(f, g)$ reduces to $-2 x_{i+s} x_{i+t}$ with respect to $\alpha$, and by Lemma 2.4 it reduces to

$$
(-1)^{\frac{t-s}{2}} 2 x_{i+\frac{s+t}{2}}^{2} \quad \text { if } \quad s+t \equiv 0 \bmod 2
$$


or it reduces to

$$
(-1)^{\frac{t-s-1}{2}} 2 x_{i+\frac{s+t-1}{2}} x_{i+\frac{s+t+1}{2}} \quad \text { if } \quad s+t \equiv 1 \bmod 2 .
$$

By hypothesis on indices $s, s^{\prime}, t, t^{\prime}$ we have

$$
\begin{gathered}
1 \leq i \leq m+n-5 \\
2 \leq s \leq m-1 \text { and } 1 \leq t \leq n-1 \\
1 \leq s^{\prime} \leq m-1 \text { and } 2 \leq t^{\prime} \leq n-1, \\
5 \leq i+s+t \leq m+n-1,
\end{gathered}
$$

if $i=m+n-4$ or $i+s+t=4$, the only possible permanents with same leading terms are equal. If $s+t \equiv 0 \bmod 2$, then

$$
3=1+\frac{2+2}{2} \leq i+\frac{s+t}{2} \leq \frac{m+n-5+m+n-1}{2}=m+n-3,
$$

so $x_{i+\frac{s+t}{2}}^{2}$ is a monomial of type 4 . If $s+t \equiv 1 \bmod 2$ then

$3=1+\frac{2+3-1}{2} \leq i+\frac{s+t-1}{2} \leq \frac{m+n-6+m+n-1-1}{2}=m+n-4$,

In fact $s+t$ must be odd but it cannot be 3 because in this case the only possible permanent with leading terms $x_{1} x_{3}$ is $x_{1} x_{3}+x_{2}^{2}$. It follows $x_{i+\frac{s+t-1}{2}} x_{i+\frac{s+t+1}{2}}$ is a monomial of type 2 . Hence, in any case $S(f, g)$ reduces to zero with respect to $G$.

By Lemma 2.5 and Corollary 2.6 the $S$-polynomial of two permanents reduces to zero whenever the leading terms of polynomials are different.

Now it remains to prove that $S(f, g)$ reduces to zero with respect to $G$ when $f$ is a permanent and $g$ is a monomial. As before,

$$
f=x_{i} x_{i+s+t}+x_{i+s} x_{i+t}
$$

where $i=1, \ldots, m+n-3, s=1, \ldots, m-1, t=1, \ldots, n-1$ with $i+s+t=$ $3, \ldots m+n-1$.

First of all we consider $g=x_{j} x_{j+1}$ a monomial of type 2. It is impossible that $i n(f)=x_{j} x_{j+1}$, so the only possibility is that $i n(f)$ and $g$ have one factor in common.

If $i=j$ then $S(f, g)=x_{i+1} x_{i+s} x_{i+t}$. As $3 \leq i=j \leq m+n-4$, if $s=t=1$ then $S(f, g)$ is a monomial of type 4 . Otherwise $6 \leq i+s+t \leq m+n-1$ and by Lemma 2.4 for $x_{i+s} x_{i+t}$, it reduces to a multiple of

$$
\pm x_{i+\frac{s+t}{2}}^{2} \quad \text { if } \quad s+t \equiv 0 \bmod 2,
$$

or it reduces to a multiple of

$$
\pm x_{i+\frac{s+t-1}{2}} x_{i+\frac{s+t+1}{2}} \quad \text { if } \quad s+t \equiv 1 \bmod 2 .
$$


We observe that, if $s+t \equiv 0 \bmod 2$, then

$$
5=3+\frac{2+2}{2} \leq i+\frac{s+t}{2} \leq \frac{m+n-5+m+n-1}{2}=m+n-3,
$$

so $x_{i+\frac{s+t}{2}}^{2}$ is a monomial of type 4. However, if $i=m+n-4$ and $i+s+t=$ $m+n-1$, necessarily $i+s=m+n-2$ and $i+t=m+n-3$ and so it is a monomial of type 2 . If $s+t \equiv 1 \bmod 2$ then

$4=3+\frac{2+1-1}{2} \leq i+\frac{s+t-1}{2} \leq \frac{m+n-4+m+n-1-1}{2}=m+n-3$,

so $x_{i+\frac{s+t-1}{2}} x_{i+\frac{s+t+1}{2}}$ is a monomial of type 2 . Hence, in any case it reduces to zero with respect to $G$. It is simple to see that also in the cases $x_{i}=x_{j+1}$, $x_{i+s+t}=x_{j}$ or $x_{i+s+t}=x_{j+1}$ the $S$-polynomials reduce to zero with respect to $G$.

Now, let $g=x_{2}^{2} x_{3}$. It is impossible that $x_{i} x_{i+s+t}=x_{2} x_{3}$ so the only possibilities are $x_{i}=x_{2}, x_{i}=x_{3}$ or $x_{i+s+t}=x_{3}$.

If $i=2$ then $S\left(f, x_{2}^{2} x_{3}\right)=x_{2} x_{3} x_{2+s} x_{2+t}$. Now we can apply the Lemma 2.4 As $m \geq 3$ and $n \geq 5$, then

$$
\begin{gathered}
\frac{m+n}{2}<m+n-3, \\
3 \leq 2+\frac{s+t}{2} \leq \frac{m+n}{2}<m+n-3 \text { if } s+t \equiv 0 \bmod 2, \\
3 \leq i+\frac{s+t-1}{2} \leq \frac{m+n}{2}<m+n-3<m+n-2 \text { if } s+t \equiv 1 \bmod 2 .
\end{gathered}
$$

These arguments show that $S(f, g)$ reduces to zero with respect to $G$.

If $i=3$, then $S\left(f, x_{2}^{2} x_{3}\right)=x_{2}^{2} x_{3+s} x_{3+t}$. The same argument shows that it reduces to zero with respect to $G$.

If $i+s+t=3$ then $i=s=t=1$ so $S\left(f, x_{2}^{2} x_{3}\right)=x_{2}^{4}$ that reduces to zero with respect to $G$.

By symmetry, if $g=x_{m+n-3} x_{m+n-2}^{2}$ then $S(f, g)$ reduces to zero with respect to $G$.

If $g$ is a monomial of type 4 , then $S(f, g)=x_{j} x_{i+s} x_{i+t}$ not only in the case $i=j$ but also in the case $i+s+t=j$. In both cases, by Lemma 2.4 and previous arguments, it reduces to zero with respect to $G$.

It remains to consider $g$ a monomial of type 5 . If $g=x_{2}^{4}$ then, in order to have a nontrivial $S$-polynomial, we can have only $x_{i}=x_{2}$ so $S(f, g)=x_{2}^{3} x_{2+s} x_{2+t}$, which reduces to zero by Lemma 2.4. Finally, we see that if $g=x_{m+n-2}^{4}$ then, in order to have a nontrivial $S$-polynomial, the only possibility is $x_{i+s+t}=x_{m+n-2}$ and so $S(f, g)=x_{m+n-2}^{3} x_{m+n-2-t} x_{m+n-2-s}$ reduces to zero with respect to $G$, by Lemma 2.4 . 


\section{Minimal primes and minimal components of $P_{2}(M)$}

For the rest of this paper we set:

1. $r=m+n-2$, and to avoid the trivial case, we assume $r \geq 3$;

2. $P_{1}=\left(x_{1}, \ldots, x_{r}\right)$ and $P_{2}=\left(x_{2}, \ldots, x_{r+1}\right)$;

3. for all $r \geq 3$

$$
\begin{aligned}
Q_{1}= & \left(x_{1}, \ldots, x_{r-3}, x_{r-2}^{2}, x_{r-2} x_{r-1}, x_{r-2} x_{r}, x_{r-2} x_{r+1}+x_{r-1} x_{r},\right. \\
& \left.x_{r-1}^{2}, x_{r-1} x_{r+1}+x_{r}^{2}\right), \\
Q_{2}= & \left(x_{5}, \ldots, x_{r+1}, x_{1} x_{3}+x_{2}^{2}, x_{1} x_{4}+x_{2} x_{3}, x_{2} x_{4}, x_{3}^{2}, x_{3} x_{4}, x_{4}^{2}\right) .
\end{aligned}
$$

We now prove that $P_{1}$ and $P_{2}$ are the minimal primes of $P_{2}(M)$ and $Q_{1}$ and $Q_{2}$ are the corresponding minimal components.

Proposition 3.1. With $M$ an $m \times n$ Hankel matrix as in (1), $m+n \geq 5$, the prime ideals of $R$ minimal over $P_{2}(M)$ are

$$
P_{1}=\left(x_{1}, x_{2}, \ldots, x_{m+n-2}\right) \text { and } P_{2}=\left(x_{2}, x_{3}, \ldots, x_{m+n-1}\right) .
$$

Proof. Let $P$ be a minimal prime over $P_{2}(M)$. By Lemma 2.1, $x_{1} x_{3}^{2} \in$ $P_{2}(M)$. Then

$$
x_{1} x_{3}^{2} \in P \Rightarrow x_{1} \in P \text { or } x_{3} \in P .
$$

We suppose that $x_{1} \in P$. Since $x_{i}^{2}+x_{i-1} x_{i+1} \in P_{2}(M) \subseteq P$ for all $i=$ $2, \ldots, m+n-2$ and $P$ is prime, by induction on $i$ we have that $x_{1}, x_{2}, \ldots$, $x_{m+n-2}$ are all elements of $P$. Therefore

$$
P_{2}(M) \subseteq\left(x_{1}, \ldots, x_{m+n-2}\right) \subseteq P,
$$

and by minimality of $P$ we have that one of the minimal primes is

$$
P=P_{1}=\left(x_{1}, \ldots, x_{m+n-2}\right) .
$$

Now, if $x_{1} \notin P$ then $x_{3} \in P$. We note that

$$
x_{1} x_{i+j-1}+x_{i} x_{j} \in P_{2}(M) \subseteq P \text { for all } i=2, \ldots, m \text { and } j=2, \ldots, n .
$$

Then the case $i=j=2$ implies that

$$
x_{1} x_{3}+x_{2}^{2} \in P \text { and } x_{3} \in P \text { so that } x_{2} \in P .
$$

Suppose we have proved that $x_{2}, x_{3}, \ldots, x_{r} \in P$ for some $r \geq 3$. Then by choosing $i \in\{2, \ldots, m\}, j \in\{2, \ldots, n\}$ such that $i+j-1=r+1$, necessarily $i, j \leq r$, so that

$$
x_{1} x_{r+1}+x_{i} x_{j} \in P_{2}(M) \subseteq P
$$


implies that $x_{r+1} \in P$. Therefore

$$
P_{2}(M) \subseteq\left(x_{2}, \ldots, x_{m+n-1}\right) \subseteq P,
$$

and by minimality of $P$ we have that the only other minimal prime over $P_{2}(M)$ is

$$
P=P_{2}=\left(x_{2}, \ldots, x_{m+n-1}\right) .
$$

Proposition 3.2. The ideals $Q_{1}$ and $Q_{2}$ are respectively primary to $P_{1}$ and $P_{2}$.

Proof. By symmetry, it is sufficient to prove that $Q_{1}$ is primary to $P_{1}$. By the structure of $Q_{1}$, it is sufficient to prove the assertion only in the case $r=3$. Set $A=\left\{x_{1}^{2}, x_{1} x_{2}, x_{1} x_{3}, x_{2}^{2}, x_{1} x_{4}+x_{2} x_{3}, x_{2} x_{4}+x_{3}^{2}\right\}$. We establish the following:

1. the degree lexicographic monomial ordering $x_{1}>x_{2}>x_{3}$ and $x_{4}$ treated as a constant;

2. $P_{2}(M) \subseteq(A)$;

3. $(A) \subseteq P_{2}(M)_{\left(x_{1}, x_{2}, x_{3}\right)} \cap K\left[x_{1}, x_{2}, x_{3}, x_{4}\right]$;

4. all $S$-polynomials of elements in $A$ reduce to zero with respect to $A$;

5. the leading coefficients of elements of $A$ are elements of $K$ (do not involve $x_{4}$ ).

Then, by arguments in the proof of Proposition 3.6 of Gianni, Trager and Zacharias [6], we have that $(A)=P_{2}(M)_{\left(x_{1}, x_{2}, x_{3}\right)} \cap K\left[x_{1}, x_{2}, x_{3}, x_{4}\right]$ is the $\left(x_{1}, x_{2}, x_{3}\right)$-primary component.

Clearly $P_{2}(M) \subseteq(A)$.

Moreover, it easy to prove that $(A) \subseteq P_{2}(M)_{\left(x_{1}, x_{2}, x_{3}\right)} \cap K\left[x_{1}, x_{2}, x_{3}, x_{4}\right]$. In fact, by Lemma 2.1 the monomials $x_{1} x_{2} x_{4}, x_{1} x_{3} x_{4}, x_{2}^{2} x_{4}$, are in $P_{2}(M)$ and so, as $x_{4}$ is units in $R_{\left(x_{1}, x_{2}, x_{3}\right)}$, we have that $x_{1} x_{2}, x_{1} x_{3}, x_{2}^{2}$ are in $P_{2}(M)_{\left(x_{1}, x_{2}, x_{3}\right)}$. Now, we notice that $x_{1}^{2} x_{4}=x_{1}\left(x_{1} x_{4}+x_{2} x_{3}\right)-\left(x_{1} x_{2}\right) x_{3}$ is in $P_{2}(M)_{\left(x_{1}, x_{2}, x_{3}\right)}$ and so, as $x_{4}$ is units in $R_{\left(x_{1}, x_{2}, x_{3}\right)}$ we have that $x_{1}^{2}$ is in $P_{2}(M)_{\left(x_{1}, x_{2}, x_{3}\right)}$.

Finally we prove that the $S$-polynomials of elements in $A$ reduce to zero with respect to $A$. As the $S$-polynomial of two monomials reduces to zero, it is sufficient to prove that $S(f, g)$ reduces to zero when $f$ is a permanent. For example,

$$
S\left(x_{1} x_{4}+x_{2} x_{3}, x_{2} x_{4}+x_{3}^{2}\right)=x_{2}^{2} x_{3}-x_{1} x_{3}^{2} \stackrel{x_{2}^{2}}{\longrightarrow}-x_{1} x_{3}^{2} \stackrel{x_{1} x_{3}}{\longrightarrow} 0 .
$$

The others are analogous. 


\section{A primary decomposition of $P_{2}(M)$}

In this section we find a redundant primary decomposition of the ideal $P_{2}(M)$. We start by identifying what it will be the embedded component in the cases in which this will be present.

Proposition 4.1. The ideal $J=P_{2}(M)+\left(x_{1}^{2}, x_{r+1}^{2}\right)$ is primary to $\left(x_{1}, \ldots, x_{r+1}\right)$.

Proof. To prove that the ideal $J$ is primary to $\left(x_{1}, x_{2}, \ldots, x_{r+1}\right)$, we compute $\sqrt{J}$. Clearly $x_{1}, x_{r+1} \in \sqrt{J}, x_{1} x_{3}+x_{2}^{2} \in J \subseteq \sqrt{J}$ so $x_{2}^{2} \in \sqrt{J}$ so $x_{2} \in \sqrt{J}$. Say $x_{1}, \ldots, x_{i} \in \sqrt{J}, i<r$. We see that $x_{i} x_{i+2}+x_{i+1}^{2} \in J \subseteq \sqrt{J}$ so $x_{i+1}^{2} \in$ $\sqrt{J}$ and so $x_{i+1} \in \sqrt{J}$. So $J \subseteq\left(x_{1}, \ldots, x_{r+1}\right) \subseteq \sqrt{J}$. But $\left(x_{1}, \ldots, x_{r+1}\right)$ is maximal in $R=K\left[x_{1}, \ldots, x_{r+1}\right]$, so $J$ is primary.

Now we recall a fact whose proof is folklore.

Fact 4.2. In an Noetherian ring $R$, for all ideals $I$ and for element $x \notin \sqrt{I}$, there exists an integer $n$ such that

$$
\left(I: x^{n}\right)=\left(I: x^{n+1}\right)
$$

and then

$$
I=\left(I: x^{n}\right) \cap\left(I+\left(x^{n}\right)\right) .
$$

Theorem 4.3. Let $M$ be an $m \times n$ Hankel matrix in $K\left[x_{1}, x_{2}, \ldots, x_{m+n-1}\right]$ as in (11). Let $P_{2}(M)$ be the ideal generated by the $2 \times 2$ permanents of $M$. Then a possibly redundant primary decomposition of $P_{2}(M)$ is $P_{2}(M)=$ $Q_{1} \cap Q_{2} \cap J$.

Proof. We show that

1. $Q_{1}=\left(P_{2}(M): x_{r+1}^{2}\right)=\left(P_{2}(M): x_{r+1}^{3}\right)$,

2. $Q_{2}=\left(P_{2}(M)+\left(x_{r+1}^{2}\right)\right): x_{1}^{2}=\left(P_{2}(M)+\left(x_{r+1}^{2}\right)\right): x_{1}^{3}$,

and so by Fact 4.2 we can assert that

$$
P_{2}(M)=Q_{1} \cap Q_{2} \cap J
$$

is a primary decomposition of $P_{2}(M)$.

1. (a) First of all we see that $x_{r+1}^{2} Q_{1} \subseteq P_{2}(M)$. As $x_{r-2} x_{r+1}+x_{r-1} x_{r}$, and $x_{r-1} x_{r+1}+x_{r}^{2}$ are two permanents, it is clear that $\left(x_{r-2} x_{r+1}+\right.$ $\left.x_{r-1} x_{r}\right) x_{r+1}^{2},\left(x_{r-1} x_{r+1}+x_{r}^{2}\right) x_{r+1}^{2}$ are in $P_{2}(M)$. By Lemma 2.1] $x_{r-2}^{2} x_{r+1}^{2}, x_{r-2} x_{r-1} x_{r+1}^{2}, x_{r-2} x_{r} x_{r+1}^{2}$ and $x_{r-1}^{2} x_{r+1}^{2}$ are in $P_{2}(M)$. If $r \geq 4$ and $i=1, \ldots, r-3$ then $x_{i} x_{r+1}^{2} \in P_{2}(M)$. In fact as $\left(x_{i} x_{r+1}+x_{s} x_{t}\right)$ with $s, t$ such that $s+t=i+r+1$ is a permanent and as, by Lemma 2.1. $x_{s} x_{t} x_{r+1}$ is in $P_{2}(M)$ we have that $x_{i} x_{r+1}^{2}=x_{r+1}\left(x_{i} x_{r+1}+x_{s} x_{t}\right)-x_{s} x_{t} x_{r+1}$ is in $P_{2}(M)$. So we have $Q_{1} \subseteq\left(P_{2}(M): x_{r+1}^{2}\right)$. But we know that $P_{2}(M) \subseteq Q_{1}$ and $Q_{1}$ is primary, so $Q_{1} \subseteq\left(P_{2}(M): x_{r+1}^{2}\right) \subseteq\left(Q_{1}: x_{r+1}^{2}\right)=Q_{1}$. Then $\left(P_{2}(M): x_{r+1}^{2}\right)=Q_{1}$. 
(b) For all $y \notin \sqrt{Q_{1}}$ we have $Q_{1} x_{r+1}^{2} y \subseteq P_{2}(M) \subseteq Q_{1}$ so $Q_{1}=$ $\left(P_{2}(M): x_{r+1}^{2} y\right)$ and in particular we have $Q_{1}=\left(P_{2}(M)\right.$ : $\left.x_{r+1}^{2}\right)=\left(P_{2}(M): x_{r+1}^{3}\right)$.

2. (a) By symmetry, $x_{1}^{2} Q_{2} \subseteq P_{2}(M)$. So we have $Q_{2} \subseteq\left(P_{2}(M)+\right.$ $\left.\left(x_{r+1}^{2}\right)\right): x_{1}^{2}$. But $P_{2}(M)+\left(x_{r+1}^{2}\right) \subseteq Q_{2}$ and $Q_{2}$ is primary, so $Q_{2} \subseteq\left(\left(P_{2}(M)+\left(x_{r+1}^{2}\right)\right): x_{1}^{2}\right) \subseteq\left(Q_{2}: x_{1}^{2}\right)=Q_{2}$. Then $\left(\left(P_{2}(M)+\left(x_{r+1}^{2}\right)\right): x_{1}^{2}\right)=Q_{2}$.

(b) For all $y \notin \sqrt{Q_{2}}$ we have $Q_{2} x_{1}^{2} y \subseteq P_{2}(M)+\left(x_{r+1}^{2}\right) \subseteq Q_{2}$ so $Q_{2}=\left(\left(P_{2}(M)+\left(x_{r+1}^{2}\right)\right): x_{1}^{2} y\right)$ and in particular we have $Q_{2}=$ $\left(\left(P_{2}(M)+\left(x_{r+1}^{2}\right)\right): x_{1}^{2}\right)=\left(\left(P_{2}(M)+\left(x_{r+1}^{2}\right)\right): x_{1}^{3}\right)$.

Observation 4.4. Whereas $Q_{1}$ and $Q_{2}$ are never redundant, $J$ may be redundant, but only in finitely many cases. We describe precisely what happens in the next Sections.

\section{When there is an embedded component}

Proposition 5.1. Let $M$ be an $m \times n$ Hankel matrix as in (1).

1. If $m=2$ and $n \geq 4$, then $\left(x_{1}, x_{2}, \ldots, x_{n+1}\right)$ is an associated prime of $P_{2}(M)$.

2. If $m \geq 3$ and $m+n-1 \geq 9$, then $\left(x_{1}, \ldots, x_{m+n-1}\right)$ is an associated prime of $P_{2}(M)$.

Proof. By definition, an ideal $J$ is an associated prime to $P_{2}(M)$ if there exists $\alpha \in K\left[x_{1}, \ldots, x_{m+n-1}\right]$ such that

$$
J=\left(P_{2}(M): \alpha\right) .
$$

1. Let

$$
M=\left[\begin{array}{ccccccc}
x_{1} & x_{2} & x_{3} & x_{4} & \ldots & x_{n-1} & x_{n} \\
x_{2} & x_{3} & x_{4} & x_{5} & \ldots & x_{n} & x_{n+1}
\end{array}\right],
$$

and for all $i, j$ with $i<j, \quad i=2, \ldots, n-2, \quad j=4, \ldots, n$,

$$
\alpha=x_{i} x_{j}
$$

Then, by Lemma 2.1] we have

$$
\left(x_{1}, x_{2}, \ldots, x_{n+1}\right) \subseteq\left(P_{2}(M): \alpha\right),
$$

and Theorem 2.7 implies that $\alpha \notin P_{2}(M)$. 
2. Now let $M$ a $m \times n$ Hankel matrix as in (11). We assume $m \geq 3$ and $m+n-1 \geq 9$. For all $j=5, \ldots,(m+n-1)-4$ set

$$
\alpha=x_{j}
$$

Then by degree count, $\alpha \notin P_{2}(M)$. So it is sufficient to prove that

$$
\alpha\left(x_{1}, \ldots, x_{m+n-1}\right) \subseteq P_{2}(M) .
$$

By Lemma 2.4, for all $i=1, \ldots, m+n-1$ and $j=5, \ldots, m+n-5$ the monomials $x_{i} x_{j}$ reduce, with respect to elements of $P_{2}(M)$, to

$$
\begin{gathered}
\pm x_{\frac{i+j}{2}}^{2} \quad \text { if } i+j \equiv 0 \bmod 2, \\
\pm x_{\frac{i+j-1}{2}} x_{\frac{i+j+1}{2}} \text { if } i+j \equiv 1 \bmod 2 .
\end{gathered}
$$

Clearly, if $i+j \equiv 0 \bmod 2$, then

$$
3 \leq \frac{i+j}{2} \leq m+n-3
$$

and if $i+j \equiv 1 \bmod 2$, then

$$
3 \leq \frac{i+j-1}{2} \leq m+n-4 .
$$

Thus, by Theorem 2.10, all monomials $x_{i} x_{j}$ are in $P_{2}(M)$.

Proposition 5.2. Let $M$ be a $3 \times 3$ Hankel matrix. Then the ideal $\left(x_{1}, \ldots, x_{5}\right)$ is an associated prime of $P_{2}(M)$.

Proof. In this case

$$
M=\left[\begin{array}{lll}
x_{1} & x_{2} & x_{3} \\
x_{2} & x_{3} & x_{4} \\
x_{3} & x_{4} & x_{5}
\end{array}\right] .
$$

Set $\alpha=x_{1} x_{3} x_{5}$. Then by Lemma 2.1 and 2.2

$$
\left(x_{1}, \ldots, x_{5}\right) \subseteq\left(P_{2}(M): \alpha\right),
$$

and by Proposition $2.8 x_{1} x_{3} x_{5} \notin P_{2}(M)$.

Proposition 5.3. Let $M$ be a $3 \times 4$ Hankel matrix. Then the ideal $\left(x_{1}, \ldots, x_{6}\right)$ is an associated prime of $P_{2}(M)$. 
Proof. As in previous propositions we see that if

$$
M=\left[\begin{array}{llll}
x_{1} & x_{2} & x_{3} & x_{4} \\
x_{2} & x_{3} & x_{4} & x_{5} \\
x_{3} & x_{4} & x_{5} & x_{6}
\end{array}\right],
$$

and if $\alpha=x_{2} x_{5}$ or $\alpha=x_{3} x_{4}$ then, Lemma 2.1 implies that

$$
\left(x_{1}, \ldots, x_{6}\right) \subseteq\left(P_{2}(M): \alpha\right) .
$$

Proposition 2.9 shows that $\alpha \notin P_{2}(M)$.

Proposition 5.4. Let $M$ be a $4 \times 4$ Hankel matrix. Then $\left(x_{1}, \ldots, x_{7}\right)$ is an associated prime of $P_{2}(M)$.

Proof. In this case we see that if

$$
M=\left[\begin{array}{llll}
x_{1} & x_{2} & x_{3} & x_{4} \\
x_{2} & x_{3} & x_{4} & x_{5} \\
x_{3} & x_{4} & x_{5} & x_{6} \\
x_{4} & x_{5} & x_{6} & x_{7}
\end{array}\right],
$$

and if $\alpha$ is any of $x_{2} x_{5}, x_{3} x_{4}, x_{3} x_{6}$ or $x_{4} x_{5}$ then Lemma 2.1 implies that

$$
\left(x_{1}, \ldots, x_{7}\right) \subseteq\left(P_{2}(M): \alpha\right) .
$$

The Proposition 2.9 shows that $\alpha \notin P_{2}(M)$.

\section{When there are only the minimal components}

We have mentioned that in a few cases the primary decomposition of $P_{2}(M)$ admits only the two minimal components. In this section we describe all such cases. Throughout we will use the following easy facts (for the proofs see [2] and [1]).

Fact 6.1. For all ideal $I, J, K$ then

$$
(I+J) \cap(I+K)=I+J \cap(I+K) .
$$

Fact 6.2. Let $t$ be a variable over $R=K\left[x_{1}, \ldots, x_{m+n-1}\right]$. We impose on $R[t]$ a monomial order such that for any $f \in R[t] \backslash R$, in $(f) \notin R$. For all pairs of ideals $I, J$ in $R$ we can compute $I \cap J$ via Gröbner basis. Namely,

$$
I \cap J=(I t R[t]+J(t-1) R[t]) \cap R .
$$

Proposition 6.3. Let $M$ be a $2 \times 3$ Hankel matrix, then the primary decomposition of $M$ is $Q_{1} \cap Q_{2}$. 
Proof. It is sufficient to show that $P_{2}(M)=Q_{1} \cap Q_{2}$. Actually, we can compute the intersection of the minimal components.

$$
\begin{aligned}
& Q_{1}=\left(x_{1}^{2}, x_{1} x_{2}, x_{1} x_{3}, x_{2}^{2}, x_{1} x_{4}+x_{2} x_{3}, x_{2} x_{4}+x_{3}^{2}\right), \\
& Q_{2}=\left(x_{2} x_{4}, x_{3}^{2}, x_{3} x_{4}, x_{4}^{2}, x_{1} x_{3}+x_{2}^{2}, x_{1} x_{4}+x_{2} x_{3}\right) .
\end{aligned}
$$

By using the Fact 6.1

$$
\begin{aligned}
Q_{1} \cap Q_{2}= & \left(x_{1} x_{4}+x_{2} x_{3}, x_{2} x_{4}+x_{3}^{2}\right)+ \\
& {\left[\left(x_{1}^{2}, x_{1} x_{2}, x_{1} x_{3}, x_{2}^{2}\right) \cap\left(x_{2} x_{4}, x_{3}^{2}, x_{3} x_{4}, x_{4}^{2}, x_{1} x_{3}+x_{2}^{2}, x_{1} x_{4}+x_{2} x_{3}\right)\right] } \\
= & \left(x_{1} x_{4}+x_{2} x_{3}, x_{2} x_{4}+x_{3}^{2}, x_{1} x_{3}+x_{2}^{2}\right)+ \\
& {\left[\left(x_{1}^{2}, x_{1} x_{2}, x_{1} x_{3}, x_{2}^{2}\right) \cap\left(x_{2} x_{4}, x_{3}^{2}, x_{3} x_{4}, x_{4}^{2}, x_{1} x_{4}+x_{2} x_{3}\right)\right] } \\
= & P_{2}(M)+\left[\left(x_{1}^{2}, x_{1} x_{2}, x_{1} x_{3}, x_{2}^{2}\right) \cap\left(x_{2} x_{4}, x_{3}^{2}, x_{3} x_{4}, x_{4}^{2}, x_{1} x_{4}+x_{2} x_{3}\right)\right] .
\end{aligned}
$$

By using Fact 6.2 it is straightforward to see that the last intersection is equal to

$$
\left(x_{1}^{2} x_{4}+x_{1} x_{2} x_{3}, x_{1} x_{2} x_{4}, x_{1} x_{3}^{2}, x_{1} x_{3} x_{4}, x_{2}^{2} x_{3}, x_{2}^{2} x_{4}\right) .
$$

Clearly $x_{1}^{2} x_{4}+x_{1} x_{2} x_{3}$ is in $P_{2}(M)$. By Lemma 2.1 the monomials $x_{1} x_{2} x_{4}$, $x_{1} x_{3}^{2}, x_{1} x_{3} x_{4}, x_{2}^{2} x_{3}, x_{2}^{2} x_{4}$ are in $P_{2}(M)$, so

$$
Q_{1} \cap Q_{2}=P_{2}(M)
$$

Proposition 6.4. Let $M$ be a $3 \times 5$ Hankel matrix. Then the primary decomposition of $M$ is $Q_{1} \cap Q_{2}$.

Proof. We know that

$$
\begin{aligned}
& Q_{1}=\left(x_{1}, x_{2}, x_{3}, x_{4}^{2}, x_{4} x_{5}, x_{4} x_{6}, x_{5}^{2}, x_{4} x_{7}+x_{5} x_{6}, x_{5} x_{7}+x_{6}^{2}\right), \\
& Q_{2}=\left(x_{5}, x_{6}, x_{7}, x_{2} x_{4}, x_{3}^{2}, x_{3} x_{4}, x_{4}^{2}, x_{1} x_{3}+x_{2}^{2}, x_{1} x_{4}+x_{2} x_{3}\right) .
\end{aligned}
$$

As $\left(x_{4}^{2}, x_{4} x_{5}, x_{4} x_{6}, x_{5}^{2}, x_{4} x_{7}+x_{5} x_{6}, x_{5} x_{7}+x_{6}^{2}\right) \subseteq\left(x_{5}, x_{6}, x_{7}, x_{4}^{2}\right)$, by using the Fact 6.1, we have

$$
\begin{aligned}
Q_{1} \cap Q_{2}= & \left(x_{4}^{2}, x_{4} x_{5}, x_{4} x_{6}, x_{5}^{2}, x_{4} x_{7}+x_{5} x_{6}, x_{5} x_{7}+x_{6}^{2}\right)+\left[\left(x_{1}, x_{2}, x_{3}\right) \cap\right. \\
& \left.\left(x_{5}, x_{6}, x_{7}, x_{2} x_{4}, x_{3}^{2}, x_{3} x_{4}, x_{4}^{2}, x_{1} x_{3}+x_{2}^{2}, x_{1} x_{4}+x_{2} x_{3}\right)\right] .
\end{aligned}
$$

Moreover, as $\left(x_{2} x_{4}, x_{3}^{2}, x_{3} x_{4}, x_{1} x_{3}+x_{2}^{2}, x_{1} x_{4}+x_{2} x_{3}\right) \subseteq\left(x_{1}, x_{2}, x_{3}\right)$, by using the Fact 6.1, we have

$$
\begin{aligned}
Q_{1} \cap Q_{2}= & \left(x_{4}^{2}, x_{4} x_{5}, x_{4} x_{6}, x_{5}^{2}, x_{4} x_{7}+x_{5} x_{6}, x_{5} x_{7}+x_{6}^{2}\right)+ \\
& \left(x_{2} x_{4}, x_{3}^{2}, x_{3} x_{4}, x_{1} x_{3}+x_{2}^{2}, x_{1} x_{4}+x_{2} x_{3}\right)+ \\
& {\left[\left(x_{1}, x_{2}, x_{3}\right) \cap\left(x_{4}^{2}, x_{5}, x_{6}, x_{7}\right)\right] . }
\end{aligned}
$$


But we know that $\left(x_{1}, x_{2}, x_{3}\right) \cap\left(x_{4}^{2}, x_{5}, x_{6}, x_{7}\right)=\left(x_{1}, x_{2}, x_{3}\right) \cdot\left(x_{4}^{2}, x_{5}, x_{6}, x_{7}\right)$, so we have

$$
\begin{aligned}
Q_{1} \cap Q_{2}= & \left(x_{4}^{2}\right)+\left(x_{4} x_{5}, x_{4} x_{6}, x_{5}^{2}, x_{4} x_{7}+x_{5} x_{6}, x_{5} x_{7}+x_{6}^{2}\right)+ \\
& \left(x_{2} x_{4}, x_{3}^{2}, x_{3} x_{4}, x_{1} x_{3}+x_{2}^{2}, x_{1} x_{4}+x_{2} x_{3}\right)+\left(x_{1} x_{4}^{2}, x_{2} x_{4}^{2}, x_{3} x_{4}^{2}\right)+ \\
& \left(x_{1} x_{5}, x_{1} x_{6}, x_{1} x_{7}, x_{2} x_{5}, x_{2} x_{6}, x_{2} x_{7}, x_{3} x_{5}, x_{3} x_{6}, x_{3} x_{7}\right) .
\end{aligned}
$$

As $\left(x_{1} x_{4}^{2}, x_{2} x_{4}^{2}, x_{3} x_{4}^{2}\right) \subseteq\left(x_{4}^{2}\right)$, then

$$
\begin{aligned}
Q_{1} \cap Q_{2}= & \left(x_{1} x_{3}+x_{2}^{2}, x_{1} x_{4}+x_{2} x_{3}, x_{1} x_{5}, x_{1} x_{6}, x_{1} x_{7}, x_{2} x_{4},\right. \\
& x_{2} x_{5}, x_{2} x_{6}, x_{2} x_{7}, x_{3}^{2}, x_{3} x_{4}, x_{3} x_{5}, x_{3} x_{6}, x_{3} x_{7}, \\
& \left.x_{4}^{2}, x_{4} x_{5}, x_{4} x_{6}, x_{4} x_{7}+x_{5} x_{6}, x_{5}^{2}, x_{5} x_{7}+x_{6}^{2}\right) .
\end{aligned}
$$

Clearly $Q_{1} \cap Q_{2}$ contains $P_{2}(M)$, but it is also simple to show the opposite inclusion. It is sufficient to prove that all monomials in $Q_{1} \cap Q_{2}$ are in $P_{2}(M)$. As $x_{1} x_{2}+x_{3}^{2}, x_{1} x_{5}+x_{2} x_{4}$ and $x_{2} x_{4}+x_{3}^{2}$ are in $P_{2}(M)$, then $x_{1} x_{5}$, $x_{2} x_{4}$ and $x_{3}^{2}$ are in $P_{2}(M)$. As $x_{1} x_{6}+x_{2} x_{5}, x_{1} x_{6}+x_{3} x_{4}$ and $x_{2} x_{5}+x_{3} x_{4}$ are in $P_{2}(M)$, then $x_{1} x_{6}, x_{2} x_{5}$ and $x_{3} x_{4}$ are in $P_{2}(M)$. Now we see that $x_{2} x_{6}+x_{3} x_{5}, x_{2} x_{6}+x_{4}^{2}$ and $x_{3} x_{5}+x_{4}^{2}$ are elements of $P_{2}(M)$, which implies that $x_{2} x_{6}, x_{3} x_{5}$ and $x_{4}^{2}$ are in $P_{2}(M)$. So, as $x_{1} x_{7}+x_{3} x_{5}$ is in $P_{2}(M)$, also $x_{1} x_{7}$ is in $P_{2}(M)$. Another time we see that $x_{2} x_{7}+x_{3} x_{6}, x_{2} x_{7}+x_{4} x_{5}$ and $x_{3} x_{6}+x_{4} x_{5}$ are in $P_{2}(M)$, so $x_{2} x_{7}, x_{3} x_{6}$ and $x_{4} x_{5}$ are in $P_{2}(M)$. At the end, as $x_{3} x_{7}+x_{4} x_{6}, x_{3} x_{7}+x_{5}^{2}$ and $x_{4} x_{6}+x_{5}^{2}$ are in $P_{2}(M)$ then $x_{3} x_{7}, x_{4} x_{6}$ and $x_{5}^{2}$ are in $P_{2}(M)$ too.

Proposition 6.5. Let $M$ be a $m \times n$ Hankel matrix with $(m, n)=(3,6),(4,5)$. Then the primary decomposition of $M$ is $Q_{1} \cap Q_{2}$.

Proof. As in previous Proposition we show that $P_{2}(M)=Q_{1} \cap Q_{2}$. Clearly $Q_{1} \cap Q_{2}$ contains $P_{2}(M)$. To show the opposite inclusion we compute the intersection between $Q_{1}$ and $Q_{2}$. In this case we have

$$
\begin{aligned}
& Q_{1}=\left(x_{1}, x_{2}, x_{3}, x_{4}, x_{5}^{2}, x_{5} x_{6}, x_{5} x_{7}, x_{6}^{2}, x_{5} x_{8}+x_{6} x_{7}, x_{6} x_{8}+x_{7}^{2}\right), \\
& Q_{2}=\left(x_{5}, x_{6}, x_{7}, x_{8}, x_{2} x_{4}, x_{3}^{2}, x_{3} x_{4}, x_{4}^{2}, x_{1} x_{3}+x_{2}^{2}, x_{1} x_{4}+x_{2} x_{3}\right) .
\end{aligned}
$$

By Fact 6.1 it is simple to see that

$$
\begin{aligned}
Q_{1} \cap Q_{2}= & \left(x_{1} x_{3}+x_{2}^{2}, x_{1} x_{4}+x_{2} x_{3}, x_{2} x_{4}, x_{3}^{2}, x_{3} x_{4}, x_{4}^{2}, x_{5}^{2}, x_{5} x_{6}, x_{5} x_{7},\right. \\
& \left.x_{5} x_{8}+x_{6} x_{7}, x_{6}^{2}, x_{6} x_{8}+x_{7}^{2}\right)+\left(x_{1}, x_{2}, x_{3}, x_{4}\right) \cdot\left(x_{5}, x_{6}, x_{7}, x_{8}\right) .
\end{aligned}
$$

After computing $\left(x_{1}, x_{2}, x_{3}, x_{4}\right) \cdot\left(x_{5}, x_{6}, x_{7}, x_{8}\right)$ we have

$$
\begin{aligned}
Q_{1} \cap Q_{2}= & \left(x_{1} x_{3}+x_{2}^{2}, x_{1} x_{4}+x_{2} x_{3}, x_{1} x_{5}, x_{1} x_{6}, x_{1} x_{7}, x_{1} x_{8}, x_{2} x_{4}, x_{2} x_{5},\right. \\
& x_{2} x_{6}, x_{2} x_{7}, x_{2} x_{8}, x_{3}^{2}, x_{3} x_{4}, x_{3} x_{5}, x_{3} x_{6}, x_{3} x_{7}, x_{3} x_{8}, x_{4}^{2}, x_{4} x_{5} \\
& \left.x_{4} x_{6}, x_{4} x_{7}, x_{4} x_{8}, x_{5}^{2}, x_{5} x_{6}, x_{5} x_{7}, x_{5} x_{8}+x_{6} x_{7}, x_{6}^{2}, x_{6} x_{8}+x_{7}^{2}\right)
\end{aligned}
$$

It remains to prove that all monomials in $Q_{1} \cap Q_{2}$ are in $P_{2}(M)$ too. By Lemma 2.4 it is sufficient to prove that $x_{3}^{2}, x_{3} x_{4}, x_{4}^{2}, x_{4} x_{5}, x_{5}^{2}, x_{5} x_{6}, x_{6}^{2}$ are in $P_{2}(M)$. But these are in $P_{2}(M)$ by Theorem 2.10 


\section{Acknowledgments}

The first author thanks the Ph.D program of the University of L'Aquila for support and the New Mexico State University for hospitality.

\section{References}

[1] W. W. Adams and P. Loustaunau. An Introduction to Gröbner Bases. Number 3 in Graduate Studies in Math. ams, New York, 1994.

[2] M. F. Atiyah and J. G. MacDonald. Introduction to Commutative Algebra. Addison-Wesley, Reading, Massachusetts, 1969.

[3] G. P. M. Binet. Mmoire sur un systme de formules analytiques, et leur application des considrations gomtriques. Journal de l'cole Polytechnique. Paris, 9:280-302, 1812.

[4] L. A. Cauchy. Mmoire sur les fonctions qui ne peuvent obtenir que deux valeurs gales et de signes contraires par suite des transpositions opres entre les variables qu'elles renferment. Journal de l'cole Polytechnique. Paris, 10:29-112, 1812.

[5] A. Conca. Straightening law and powers of determinantal ideals of Hankel matrices. Adv. Math., 138:n2, 236-292, 1998.

[6] P. Gianni, B. Trager, and G. Zacharias. Gröbner bases and primary decomposition of polynomial ideals. J. Symb. Comput., 6(2-3):149-167, 1988 .

[7] S.-J. Hu and M.-C. Kang. Efficient generation of the ring of invariants. J. Algebra, 180:341-363, 1996.

[8] G. A. Kirkup. Minimal primes over permanental ideals, 2005. available at http://arxiv.org/abs/math.AC/0510025.

[9] R. C. Laubenbacher and I. Swanson. Permanental ideals. Journal of Symbolic Computation, 30(2):195-205, 2000.

[10] M. Marcus and H. Minc. Permanents. Cambridge University Press, 1985 .

[11] J. Watanabe. Hankel matrices and Hankel ideals. Proc. school. SCI Tokay Uni, 32:11-21, 1997. 